\title{
Cut-Off Values of Foveal Vascular Indices in Exfoliation Glaucoma
}

This article was published in the following Dove Press journal:

Clinical Ophthalmology

\author{
Tolga Kocatürk (1D' \\ Maja Zivkovic ${ }^{2}$ \\ Volkan Dayanır ${ }^{3}$ \\ 'Department of Ophthalmology, Aydın \\ Adnan Menderes University, Aydın, \\ Turkey; ${ }^{2}$ Department of Ophthalmology, \\ Medical Faculty, University of Nis, Nis, \\ Serbia; ${ }^{3}$ Department of Ophthalmology, \\ Batıgöz Hospital, Izmir, Turkey
}

Purpose: To compare the vascular measurements obtained from fovea including foveal avascular zone (FAZ) with optical coherence tomography angiography (OCTA) between patients with exfoliation glaucoma (XFG) and healthy controls.

Methods: This cross-sectional comparative study included 54 XFG patients and 94 healthy subjects. All subjects were given a complete ophthalmological examination including visual field testing, retinal nerve fiber layer and macular ganglion cell complex analysis. OCTA was performed to evaluate the vascular features of fovea including FAZ. The measurements of vessel density (VD), perfusion density (PD) and FAZ values were segmented and calculated using the inbuilt software on OCTA. Receiver operating characteristic (ROC) analyses were performed to determine the best cut-off values to detect the disease.

Results: In the XFG group, decreased VD, PD and FAZ values were observed. ROC analysis showed an obvious separation, differentiating the patients with XFG from the healthy controls at cut-off value of 19.55 with a sensitivity (sen) of $92.3 \%$ and a specificity (spe) of 81.9\%) for VD total and 21.20 (sen:100 and spe:78.7) for VD parafoveal; 0.36 (sen:98.1 and spe:76.6) for PDtotal and 0.378 (sen:96.2and spe:84) for PDparafoveal; 0.635 (sen: 79.3 and spe:72) for FAZ circulatory index.

Conclusion: The results support the findings of decreased microvascular density on foveal region in XFG. The cut off values of the changes in the foveal parameters in patients with exfoliation may be useful in evaluation of future glaucoma management to show how far a particular exfoliative eye is positioned from an healthy eye and how close to XFG.

Keywords: exfoliation glaucoma, optical coherence tomography angiography, foveal avascular zone

\section{Introduction}

Exfoliation is an abnormally structured fibrillar material deposited extracellularly within the intraocular and extraocular tissues including systemic vascular structures in the body. ${ }^{1}$ It is also a precursor as it may lead to exfoliation glaucoma (XFG), the most common identifiable cause of open-angle glaucoma. ${ }^{2}$ Elevated intraocular pressure (IOP) is the main independent and the only modifiable risk factor; however the pathogenesis of glaucoma is still unknown. ${ }^{3}$

In its management, surgical intervention is required more frequently, as XFG is less responsive to medical therapy. Vascular compromise has long been considered as a suspect and was the focus of past investigations. ${ }^{4-6}$ Altered ocular blood flow seems to be important in the development of glaucoma, and is known to be a primary and independent risk factor for progression of the disease. ${ }^{7,8}$ Optical coherence tomography angiography (OCTA) is a new method and may offer
Aydın Adnan Menderes Universitesi, Tip FakultesiHastanesi, Goz Hast, AD, Aydin, 09100, Turkey

Tel +90533344 4I II

Fax +902562146495

Email tolgakocaturk@gmail.com 
a better understanding of the pathogenesis of glaucoma by providing a detailed map of vascular microarchitecture in fovea and FAZ,capillary-free area in central macula, with high accuracy and great reproducibility. ${ }^{9-14}$ Thus, vascular parameters were suggested as potential diagnostic tools in the following-up of the patients at risk for glaucoma.

To the best of our knowledge this is a pioneering approach for evaluating the OCTA based measurements of FAZ in XFG patients as we conducted Receiver Operating Characteristics (ROC) analysis to determine the conversion (cut-off) values from healthy subjects to XFG with high sensitivity and specificity. We aim to compare variables measured by circulatory index of FAZ with OCTA between patients with XFG and healthy controls.

\section{Methods}

A total of 148 participants were included in the study. Patients with XFG were recruited from the Eye Hospital Clinic Maja, Glaucoma Unit, Nis, Serbia between December 2017 and April 2018. This cross-sectional comparative study was approved by Institutional Ethics Committee with an approval number of Q11/10-2017.

All patients were previously diagnosed with XFG, control subjects were recruited from the outpatient clinic of the same clinic. Written informed consents were obtained from all participants included in the study. Each patient underwent a complete ophthalmic examination including best corrected visual acuity (BCVA), slit-lamp biomicroscopy, diurnal IOP measurement by Goldmann applanation tonometry (Haag-Streit, Bern, Switzerland), gonioscopy, pachymetry, optic disc assessment with a plus 78-diopter lens under dilated pupils, retinal nerve fiber analysis, and automated VF testing using Swedish Interactive Thresholding Algorithm (SITA-standard) central 24-2 Humphrey perimetry (Humphrey field analyser II 750, Carl Zeiss Meditec, Dublin, CA, USA), multicolour disc photograph, and OCTA on Zeiss AngioPlex, Cirrus HD-OCT Model 5000 instrument (Carl Zeiss Meditec, Dublin, CA).

Glaucoma patients (age $\geq 40$ years of age and male or female gender) with BCVA of $\geq 0.8$ Snellen were included in the study. All glaucoma patients were followed for at least one year in our glaucoma unit and they underwent detailed ophthalmologic examinations at least three times a year. Diagnostic criteria for XFG were adapted from the European Glaucoma Society. ${ }^{15}$ Mild glaucoma was defined as a mean deviation (MD) of $-6 \mathrm{~dB}$ or better, moderate as a MD between -6 and $-12 \mathrm{~dB}$, and severe as a $\mathrm{MD}$ of $-12 \mathrm{~dB}$ or worse. ${ }^{16}$ In both groups, only one eye of each subject was included in the study. Patients with systemic disease with ocular involvement like diabetes, neurological or other eye diseases capable of causing VF loss or optic disc deterioration (except glaucoma), opacification of ocular media, intraocular surgery, refractive errors more than \pm 2 diopters, and history of ocular trauma were excluded from the study.

Control subjects were healthy individuals (age $\geq 40$ years of age and male or female gender) with BCVA of 1.0 Snellen, normal optic discs (cup-to-disc ratio $<0.3$ with no localized defects in neuroretinal rim or margin, no splinter hemorrhage around the disc), open angles at gonioscopy, IOP $<21 \mathrm{~mm} \mathrm{Hg}$ on two different days, CCT greater than $500 \mu \mathrm{m}$ in both eyes, normal retinal nerve fiber layer (RNFL) thickness and normal standard automated perimetry (defined as a glaucoma hemifield test within normal limits and a pattern standard deviation within 95\% confidence-interval limits). Control group participants were excluded from the study if they had a family history of glaucoma or ocular hypertension, refractive error $>0.50$ diopters, or if they were on any systemic or topical medication, had any retinal pathology, corneal scars, central corneal thickness outside the normal limits, history of previous ocular surgery or trauma. ${ }^{15,17}$ Subjects with closed iridocorneal angles, history of angle closure glaucoma crisis or findings of narrow angle, evidence of secondary glaucoma ocular or systemic diseases including diabetes mellitus, obesity, or any form of retinal or eye pathology, neuro-ophthalmologic, or systemic diseases those could result in optic neuropathy, or VF defects were excluded from the study. ${ }^{15,17}$ Subjects with obstructive sleep apnea and with requirement of cuff size over 36 or below $20 \mathrm{~cm}$ were also excluded along with smokers and alcoholics were also excluded from the study.

The OCT protocol according to standardized operating procedures included Cirrus SD-OCT device (model 5000, software version 10.0, Carl Zeiss Meditec, Inc.) was used. The macular ganglion cell complex (GCC) analysis algorithm of the aforementioned device was used to process and measure the thickness of macular ganglion cell layerinner plexiform layer (GCL+IPL). The average, minimum, and six sectoral (superotemporal, superior, superonasal, inferonasal, inferior, inferotemporal) GCL+IPL thicknesses were measured from the elliptical annulus centered on the fovea. Demarcation of superior sectors was performed from nasal to temporal, thus the superonasal sector 
of GCL+IPL was marked as GC1, superior as GC2, superotemporal as GC3, while demarcation of inferior sectors was performed from temporal to nasal, marking inferotemporal as GC4, inferior GC5 and inferonasal as GC6.

OCTAwas performed by a $68 \mathrm{kHz}$ Cirrus HD-CT 5000-based Optical Micro Angiography (OMAG) prototype system. Images obtained were checked for quality (Signal Strength more than 6/10), as well as absence of artefacts.

The diameters of FAZ (horizontal, vertical, max), vessel density (VD), perfusion density (PD), area, perimeter, and circularity of FAZ were automatically segmented and calculated using the inbuilt software on OCTA Zeiss AngioPlex ${ }^{\mathrm{TM}}$. PD is defined as the total area of perfused vasculature per unit area in a region of measurement $\left(\mathrm{mm}^{2} / \mathrm{mm}^{2}\right.$, makes it unitless), and VD is defined as the total length of perfused vasculature per unit area $(\mathrm{mm} /$ $\mathrm{mm}^{2}$ or $\mathrm{mm}^{-1}$ ) in a region of measurement. AngioPlex ${ }^{\mathrm{TM}}$ evaluates VD of whole macular area (total), moreover, it is able to subdivide the macular area into three subdivisions: a central circle $(1 \mathrm{~mm})$, an inner circle $(3 \mathrm{~mm})$, and an outer circle $(6 \mathrm{~mm})$. Currently OCTA Zeiss AngioPlex allows access to $3 \times 3,6 \times 6$ and $8 \times 8$ scans. As we examined mostly moderate XFG eyes with a mean MD of $-9.57 \mathrm{~dB}$, considering that it was very important to get accurate measurement of FAZ, 3x3 scans were exclusively used as they give the better results when differentiating between healthy and moderate to severe glaucoma (Figure 1). We have analysed the dimensions of FAZ manually by measuring vertical and horizontal diameters. Also considering the phenomenon of capillary drop out we introduced the concept of maximum diameter which is defined as the longest measurable diameter within a FAZ.

\section{Statistics}

Statistical analysis was performed with the statistical package SPSS 22 for Windows. Primary data obtained were analysed by descriptive statistical methods and methods for testing hypotheses. The following descriptive statistical methods were used: measures of central tendency (mean, median) rate variability (standard deviation and variation interval) and indicators of structure expressed in

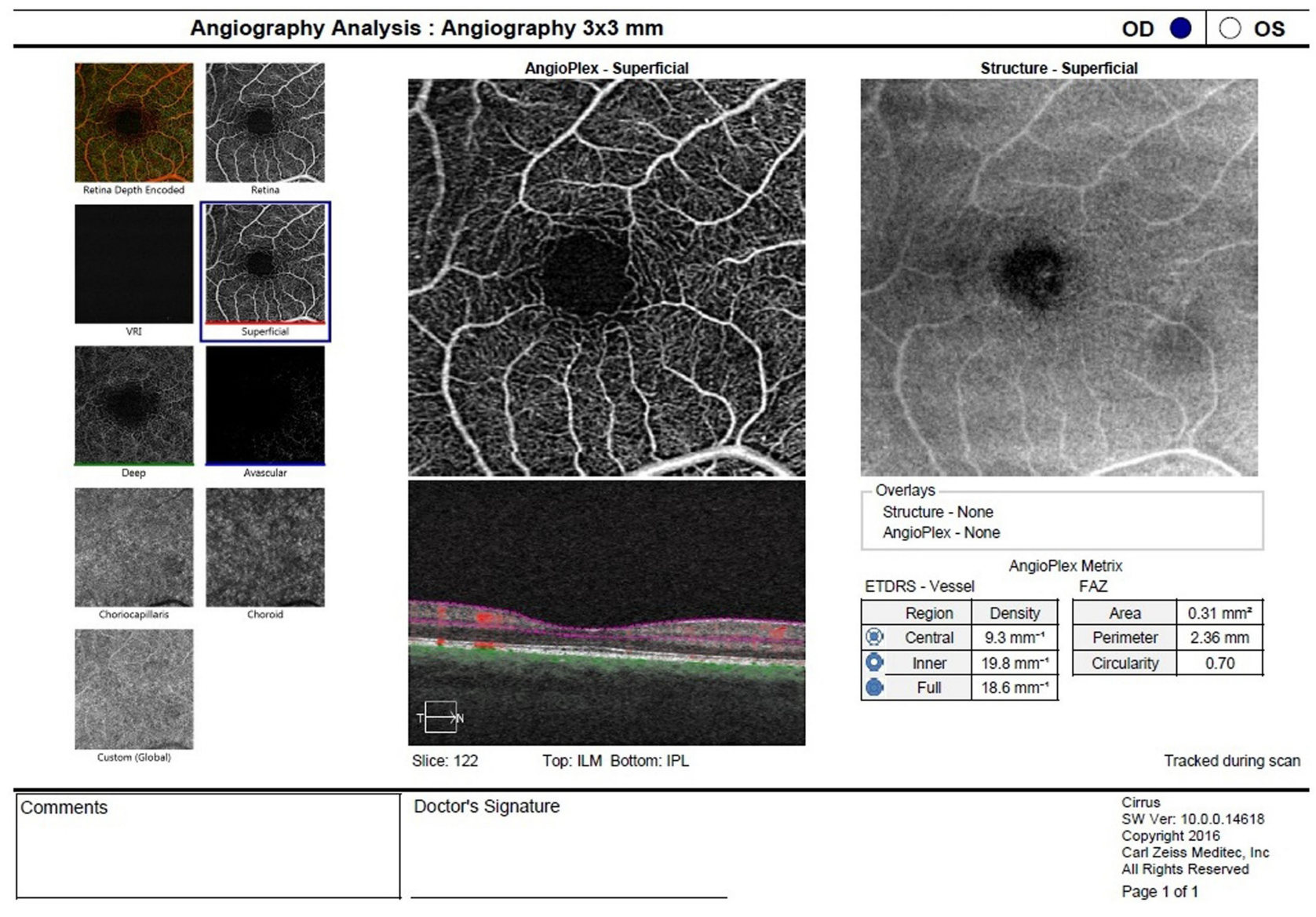

Figure I $3 \times 3$ scan of foveal avascular zone of a representative case is seen. 
percentages. Kolmogorov-Smirnov test was used to determine the normality of distribution. The difference of frequency proportions by groups is done with $\chi^{2}$ test. To test the difference of arithmetic mean between the groups the $t$-test was used. In cases where the requirements were not met, the distribution of normality and homogeneity of variance, nonparametric statistical procedures Mann-Whitney $U$-test was used. The conclusion was done at the level of statistical significance of 0.05 . Logistic regression was used to explain the relationship between the outcomes and predictors. Receiver Operating Characteristic (ROC) curve was used to determine the conversion values, sensitivity, specificity, and area under the curve for the parameters. A p-value $<0.05$ was considered as statistically significant. Correlation analysis was used to establish the relationship between two variables. The Pearson/Spearman correlation coefficient was used to measure the strength and direction of the relationships.

\section{Results}

\section{Socio-Demographic Characteristics}

Out of a total of 148 subjects, there were 54 XFG patients (Group 1), and 94 healthy individuals without glaucoma (Group 2). Groups were similar according to sex $(\mathrm{p}=0.497)$ and age distributions $(\mathrm{p}=0.065)$. Also, there were no statistically significant differences between the right and left eyes $(p=0.609)$ between group 1 and 2 (Table 1$)$. The details of the socio-demographic data are presented in Table 1.

\section{Clinical Measurements}

We analysed GCL+IPL layers of the average, minimum, and thickness on following sectors from GC1 to GC6. All mean values of all the 6 sectors GCL+IPL as well as the values of GCavg and GCmin were statistically lower in XFG patient group compared to the control group. In VF testing, Mean MD value for XFG group was statistically significant lower than the mean value for control group. Mean PSD value for XFG group was statistically significant higher than the mean value for control group. The mean value of max diameter of FAZ for XFG group was statistically significant higher than control group value. The mean values for RNFL average, superior, nasal, inferior and temporal thickness, VD -central (foveal), -inner (parafoveal) and -full (total), PD -central (foveal), -inner (parafoveal) and -full (total), area and circulatory index for XFG group were significantly lower than those for control group. There were no statistically significant differences between the mean values of central subfield thickness (CST), Angio-horizontal and -vertical, as well as perimeter between the groups (Table 2). There were no significant correlation among the variables tested in XFG group except VD total which was weakly correlated with RNFL average ( $\mathrm{p}=0.037)$ (Table 3 ).

A binary logistic model that is statistically significant $(p<0.001)$ predicted the probability of the presence of XFG, based on VD, PD and FAZ values seen in Table 4 . As an example, obtaining VD foveal as an independent variable in a binary logistic statistically significant model $(\mathrm{p}<0.001)$ and we got $\mathrm{OR}=0.69$, so we would state that the odds of having XFG were decreased by a factor of 0.69 , when the VD foveal increases by one unit (Table 4).

$\mathrm{AROC}=0.81$ for $\mathrm{VD}$ foveal, $\mathrm{AROC}=0.96$ for VD parafoveal and $\mathrm{AROC}=0.95$ for $\mathrm{VD}$ total suggested that the odds for lower VD foveal, VD parafoveal and VD total, would be greater $81 \%, 96 \%$ and $95 \%$ respectively in patient with $\mathrm{XFG}$ in comparison to the control group. $\mathrm{AROC}=0.79$ for $\mathrm{PD}$ foveal, $\mathrm{AROC}=0.96$ for $\mathrm{PD}$ parafoveal and $\mathrm{AROC}=0.95$ for $\mathrm{PD}$ total suggested that the odds for lower PD foveal, PD parafoveal and PD total, would be greater $79 \%, 96 \%$ and $95 \%$ respectively in patient with $\mathrm{XFG}$ in comparison to the control group. The similar explanation would be with $\mathrm{AROC}=0.74$ for $\mathrm{FAZ}$-area and $\mathrm{AROC}=0.78$ for FAZ-circulatory index (Table 4).

Table I The Socio-Demographic Characteristics are Similar of the Groups. There are No Statistically Significant Differences Between the Groups in Terms of Right and Left Eyes, Sex and Age Distributions

\begin{tabular}{|l|c|c|c|}
\hline & XFG Group & Control Group & P value \\
\hline Number of the Eye & 54 & 94 & \\
Eye (Left/Right) & $30(55.6 \%) / 24(44.4 \%)$ & $47(50 \%) / 47(50 \%)$ & $0.609^{\mathrm{a}}$ \\
Age (mean \pm sd) & $67.91 \pm 7.20(55-87)$ & $63.41 \pm 11.54(42-91)$ & $0.065^{\mathrm{b}}$ \\
Gender (male/female) & $22(40.7 \%) / 32(59.3 \%)$ & $44(46.8 \%) / 50(53.2 \%)$ & $0.497^{\mathrm{a}}$ \\
\hline
\end{tabular}

Notes: ${ }^{\mathrm{a}} \chi^{2}$-test, ${ }^{\mathrm{b}}$-test.

Abbreviation: XFG, exfoliation glaucoma. 
Table 2 The Structural, Vascular Parameters and Visual Field Indices are Seen. The Thicknesses of Ganglion Cell-Inner Plexiform Layers, Visual Field Testing Parameters, Optical Coherence Tomography Angiography Parameters and Retinal Nerve Fiber Layer Thicknesses of the Groups are Compared

\begin{tabular}{|c|c|c|c|c|}
\hline \multicolumn{2}{|l|}{ Parameters } & \multirow{2}{*}{$\frac{\text { XFG Group Mean } \pm \text { sd (Min-Max) }}{68.85 \pm 14.08(24-92)}$} & \multirow{2}{*}{$\begin{array}{c}\text { Control Group Meantsd (Min-Max) } \\
84.47 \pm 7.48(55-90)\end{array}$} & \multirow{2}{*}{$\begin{array}{l}\text { P value } \\
<0.001^{\mathrm{a}}\end{array}$} \\
\hline $\mathrm{GCC}(\mu \mathrm{m})$ & I & & & \\
\hline & 2 & $69.81 \pm 13.82(31-95)$ & $86.43 \pm 2.58(8 I-90)$ & $<0.001^{\mathrm{a}}$ \\
\hline & 3 & $70.52 \pm \mid 3.21(24-91)$ & $86.53 \pm 2.61(82-90)$ & $<0.00 I^{\mathrm{a}}$ \\
\hline & 4 & $69.15 \pm 12.42(24-90)$ & $86.16 \pm 12.03(70-90)$ & $<0.00 I^{\mathrm{a}}$ \\
\hline & 5 & $66.19 \pm 12.84(24-88)$ & $86.68 \pm 2.56(80-91)$ & $<0.001^{\mathrm{a}}$ \\
\hline & 6 & $68.20 \pm 14.73(22-92)$ & $86.26 \pm 2.70(82-90)$ & $<0.001^{\mathrm{a}}$ \\
\hline & Ave & $68.76 \pm 12.39(25-91)$ & $86.01 \pm 3.68(56-90)$ & $<0.001^{\mathrm{a}}$ \\
\hline & Min & $62.31 \pm 13.83(22-88)$ & $82.49 \pm 1.80(80-85)$ & $<0.001^{\mathrm{a}}$ \\
\hline CST $(\mu \mathrm{m})$ & & $260.91 \pm 34.32(163-386)$ & $258.31 \pm|8.5|(210-300)$ & $0.608^{\mathrm{a}}$ \\
\hline \multirow[t]{3}{*}{ OCTA (diameter of FAZ) $(\mu \mathrm{m})$} & Horizontal & $555.58 \pm 92.46(387-700)$ & $519.88 \pm 77.67(301-670)$ & $0.056^{\mathrm{a}}$ \\
\hline & Vertical & $489.17 \pm 121.76(257-685)$ & $494.12 \pm 82.53(302-664)$ & $0.814^{\mathrm{a}}$ \\
\hline & $\operatorname{Max}$ & $665.17 \pm 92.31(472-800)$ & $544.76 \pm 85.4 \mathrm{I}(283-796)$ & $<0.00 I^{\mathrm{a}}$ \\
\hline \multirow[t]{2}{*}{ Visual Field Testing (dB) } & $M D(d B)$ & $-9.57 \pm 10.54(-31.02--0.37)$ & $0.44 \pm 1.44(-2.69-3.63)$ & $<0.001^{\mathrm{b}}$ \\
\hline & PSD (dB) & $4.48 \pm 2.91(1.59-10.66)$ & $1.70 \pm 0.43(1.06-3.84)$ & $<0.001^{\mathrm{b}}$ \\
\hline \multirow[t]{5}{*}{ RNFL $(\mu \mathrm{m})$} & Superior & $84.44 \pm 23.14(19-128)$ & $121.65 \pm|7.1|(90-170)$ & $<0.001^{\mathrm{a}}$ \\
\hline & Nasal & $65.50 \pm 12.04(43-112)$ & $75.90 \pm 11.42(50-104)$ & $<0.001^{\mathrm{a}}$ \\
\hline & Inferior & $89.8 I \pm 24.19(42-141)$ & $|28.93 \pm| 4.6 \mid(90-180)$ & $<0.001^{\mathrm{a}}$ \\
\hline & Temporal & $58.83 \pm 12.38(39-97)$ & $67.55 \pm 10.73(45-93)$ & $<0.00 \mathrm{I}^{\mathrm{a}}$ \\
\hline & Average & $73.83 \pm 13.59(5|-| 14)$ & $98.63 \pm 10.05(80-133)$ & $<0.00 I^{\mathrm{a}}$ \\
\hline \multirow[t]{3}{*}{$\mathrm{VD}\left(\mathrm{mm}^{-1}\right)$} & Foveal & $7.50 \pm 3.43(2.0-17.0)$ & $11.40 \pm 2.93(4.1-17.8)$ & $<0.001^{\mathrm{a}}$ \\
\hline & Parafoveal & $15.74 \pm 3.34(7.2-21.1)$ & $21.97 \pm 1.60(16.3-24.6)$ & $<0.00 I^{\mathrm{a}}$ \\
\hline & Total & $15.0 \mid \pm 3.38(6.6-20.6)$ & $20.76 \pm I .64(|5|-23.7)$. & $<0.00 I^{a}$ \\
\hline \multirow[t]{3}{*}{ PD } & Foveal & $0.13 \pm 0.06(0.04-0.29)$ & $0.20 \pm 0.06(0.08-0.33)$ & $<0.001^{\mathrm{a}}$ \\
\hline & Parafoveal & $0.29 \pm 0.06(0.15-0.39)$ & $0.40 \pm 0.03(0.31-0.46)$ & $<0.001^{\mathrm{a}}$ \\
\hline & Total & $0.28 \pm 0.06(0.13-0.36)$ & $0.38 \pm 0.03(0.29-0.43)$ & $<0.001^{\mathrm{a}}$ \\
\hline FAZ-A $\left(\mathrm{mm}^{2}\right)$ & & $0.18 \pm 0.09(0.03-0.32)$ & $0.27 \pm 0.09(0.08-0.53)$ & $<0.00 I^{\mathrm{a}}$ \\
\hline FAZ-P (mm) & & $1.96 \pm 0.68(0.14-3.24)$ & $2.19 \pm 0.36(1.24-3.21)$ & $0.086^{\mathrm{a}}$ \\
\hline FAZ-Cl & & $0.55 \pm 0.16(0.22-0.94)$ & $0.66 \pm 0.10(0.31-0.83)$ & $<0.001^{\mathrm{a}}$ \\
\hline
\end{tabular}

Notes: ${ }^{\mathrm{a}}$-test, ${ }^{\mathrm{b}}$ Mann-Whitney $U$-test.

Abbreviations: XFG, exfoliation glaucoma; GCCI, superonasal sector of ganglion cell-inner plexiform layer; GCC2, superior sector of ganglion cell-inner plexiform layer; GCC3, superotemporal sector of ganglion cell-inner plexiform layer; GCC4, inferotemporal sector of ganglion cell-inner plexiform layer; GCC5, inferior sector of ganglion cell-inner plexiform layer; GCC6, inferonasal sector of ganglion cell-inner plexiform layer; GCCavg, ganglion cell-inner plexiform layer average thickness; GCCmin, ganglion cell-inner plexiform layer minimum thickness; CST, central subfield thickness; OCTA, Optical Coherence Tomography Angiography; RNFL, retinal nerve fiber layer; MD, mean deviation; PSD, pattern standard deviation; VD, Vessel density; PD, Perfusion density; FAZ, foveal avascular zone; FAZ-A, foveal avascular zone area; FAZ-P, foveal avascular zone perimeter; $\mathrm{FAZ}-\mathrm{Cl}$, foveal avascular zone circulatory index.

Corresponding $\mathrm{AROC}=0.58$ for Perimeter was not significant $(\mathrm{p}=0.184)$ and we cannot discriminate $\mathrm{XFG}$ from controls.

The ROC analysis shows a clear separation differentiating patients with XFG and healthy controls at the cut-off values for VD, PD and FAZ parameters. The sensitivity and specificity for VD total provided by this cut-off value of 19.55 was $92.3 \%$ and $81.9 \%$, respectively and for VD parafoveal by cut-off value of 21.20 was $100 \%$ and $78.7 \%$ respectively. The sensitivity and specificity for PD total provided by this cut-off value of 0.36 was $98.1 \%$ and $76.6 \%$ respectively and for PD parafoveal by cut-off value of 0.378 was $96.2 \%$ and $84 \%$ respectively. The sensitivity and specificity for FAZ area provided by this cut-off value of 0.195 was $55.2 \%$ and $84.9 \%$ respectively and for FAZ circulatory index by cut-off value of 0.635 was $79.3 \%$ and $72 \%$ respectively (Table 4 ). 
Table 3 Correlation Analyses Between Measured Parameters Within the Exfoliation Glaucoma Group Reveals No Significant Correlation Among the Variables Tested in Exfoliation Glaucoma Group Except Vessel Density-Total Which Was Weakly Correlated with Retinal Nerve Fiber Layer Thickness

\begin{tabular}{|l|c|c|c|c|}
\hline & GCC Average $\mathbf{r}^{*}(\mathbf{p})$ & RNFL Average $\mathbf{r}^{*}(\mathbf{p})$ & VF md $\mathbf{r}^{* *}(\mathbf{p})$ & VF psd $\mathbf{r}^{* *}(\mathbf{p})$ \\
\hline VD foveal & $0.03(0.813)$ & $0.23(0.098)$ & $0.13(0.557)$ & $-0.13(0.547)$ \\
VD parafoveal & $0.19(0.171)$ & $0.26(0.067)$ & $0.02(0.930)$ & $0.11(0.604)$ \\
VD total & $0.22(0.116)$ & $0.29(0.037)$ & $0.32(0.135)$ & $-0.19(0.378)$ \\
PD foveal & $-0.01(0.941)$ & $0.20(0.167)$ & $0.04(0.849)$ & $-0.05(0.836)$ \\
PD parafoveal & $0.14(0.318)$ & $0.21(0.127)$ & $-0.09(0.698)$ & $0.15(0.483)$ \\
PD total & $0.17(0.223)$ & $0.24(0.088)$ & $0.18(0.422)$ & $-0.14(0.533)$ \\
FAZ-A & $0.04(0.858)$ & $0.17(0.369)$ & $0.30(0.201)$ & $-0.16(0.509)$ \\
FAZ-P & $-0.16(0.412)$ & $-0.33(0.081)$ & $-0.03(0.892)$ & $0.22(0.350)$ \\
FAZ-Cl & $0.20(0.312)$ & $0.18(0.353)$ & $0.14(0.560)$ & $-0.10(0.688)$ \\
\hline
\end{tabular}

Notes: *Pearson's coefficient, **Spearman's coefficient.

Abbreviations: XFG, exfoliation glaucoma; GCC, ganglion cell complex; RNFL, retinal nerve fiber layer; VF md, visual field mean deviation; VF psd, visual field pattern standard deviation; VD, Vessel density; PD, Perfusion density; FAZ-A, foveal avascular zone area; FAZ-P, foveal avascular zone perimeter; FAZ-CI, foveal avascular zone circulatory index.

Table 4 Sensitivity, Specificity, Area Under the Receiver Operating Curve and Conversion Values are Seen. The ROC Analysis Shows a Clear Separation Differentiating Patients with Exfoliation Glaucoma and Healthy Controls at the Certain Cut-Off Values

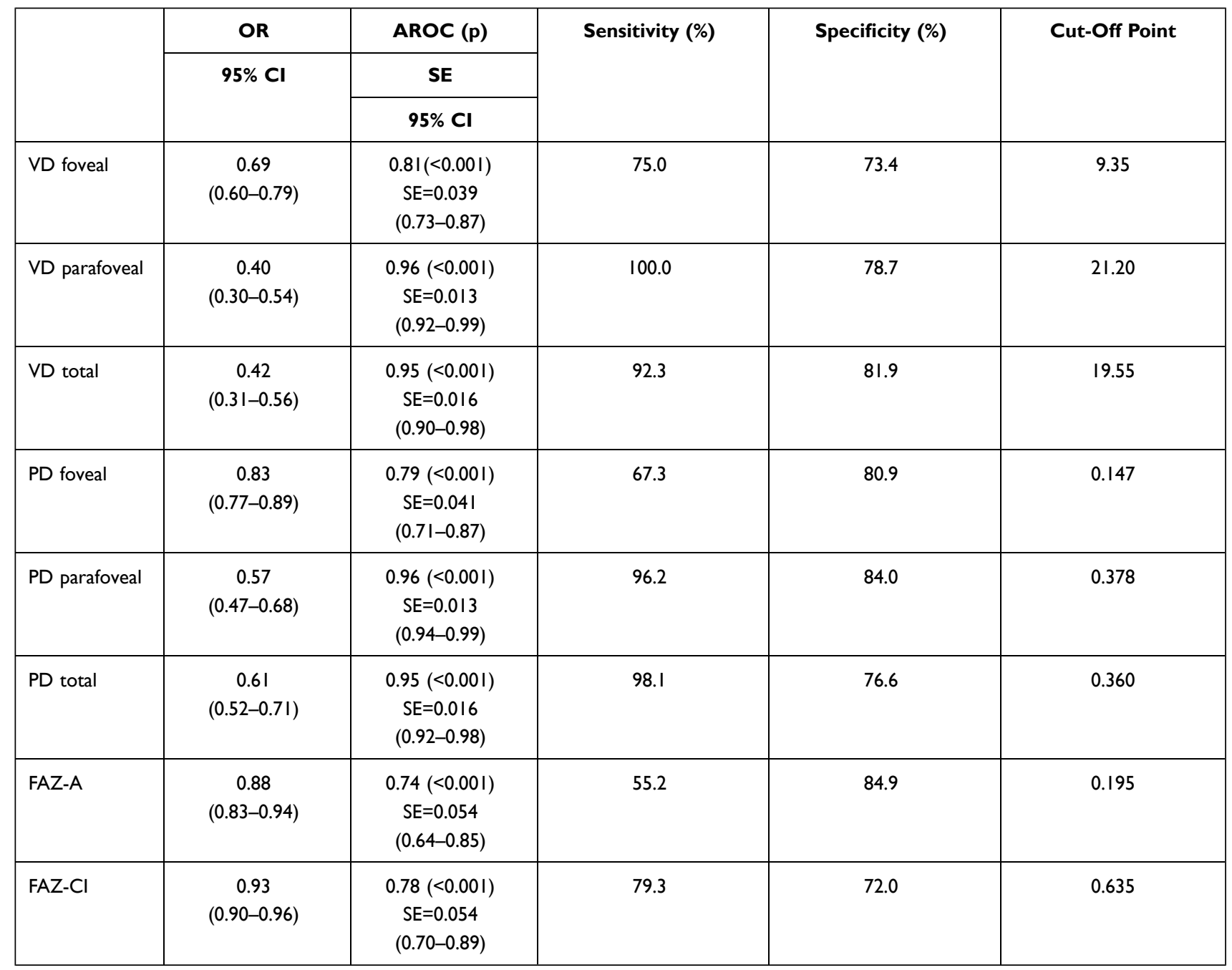

Abbreviations: XFG, exfoliation glaucoma; OR, Odds Ratio; AROC, Area under the receiver operating curve; SE, Standard Error; VD, Vessel density; PD, Perfusion density; 95\% Cl, 95\% Confidence Interval; FAZ-A, foveal avascular zone area; FAZ-Cl, foveal avascular zone circulatory index. 


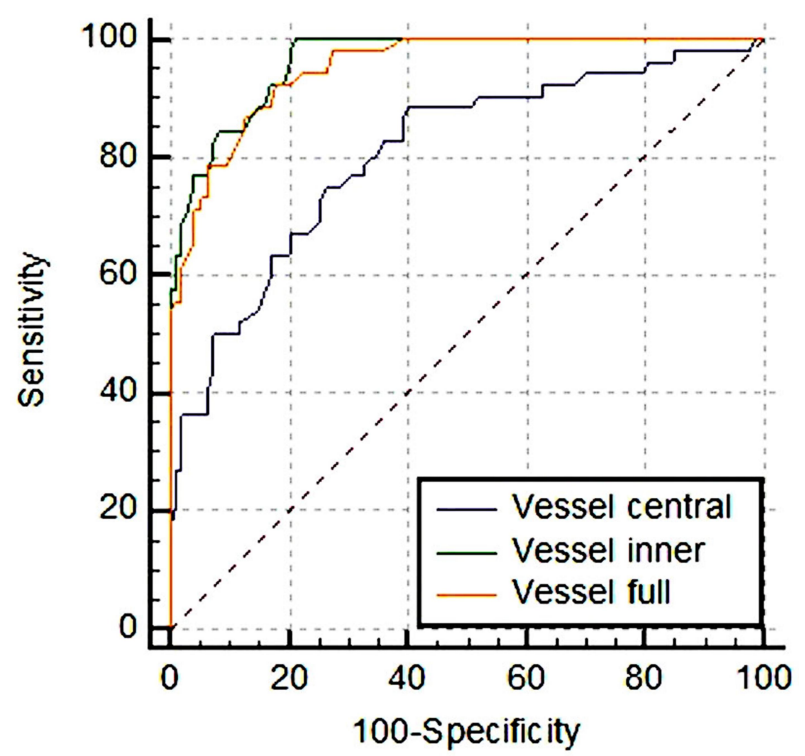

Figure $\mathbf{2}$ ROC analysis for vessel densities reveals statistically significant difference between ROC curves for VD foveal and both VD parafoveal $(p<0.001)$ and VD total $(p<0.001)$, whereas there is no statistically significant difference between ROC curves for VD parafoveal and VD total $(p=0.073)$. Both tests were highly discriminative.

There was statistically significant difference between ROC curves for VD foveal and both VD parafoveal $(p<0.001)$ and VD total $(p<0.001)$ (Figure 2). There was no statistically significant difference between the ROC curves $(p=0.073$ ) for VD parafoveal and VD total. We may conclude that both tests were highly discriminative.

There was statistically significant difference between ROC curves for PD foveal and both PD parafoveal $(\mathrm{p}<0.001)$ and PD total $(\mathrm{p}<0.001)$ (Figure 3). There was no statistically significant difference between the ROC curves $(p=0.057)$ for PD parafoveal and PD total. We may conclude that both tests were highly discriminative. There was not statistically significant difference between the ROC curves for FAZ-area and FAZ-circulatory index $(p=0.566)$, but both tests accuracy were fair.

\section{Discussion}

To the best of our knowledge this is a pioneering study to compare foveal vascular features between patients with XFG and healthy controls using OCTA, and determine certain cut-off points on the measurable parameters (indices). We demonstrated reduced VD, PD and FAZ values in XFG patients. The interesting and novel point here is the conversion (cut-off) values of those parameters in order to differentiate XFG from healthy eyes in terms of vascular indices with high sensitivity and specificity.

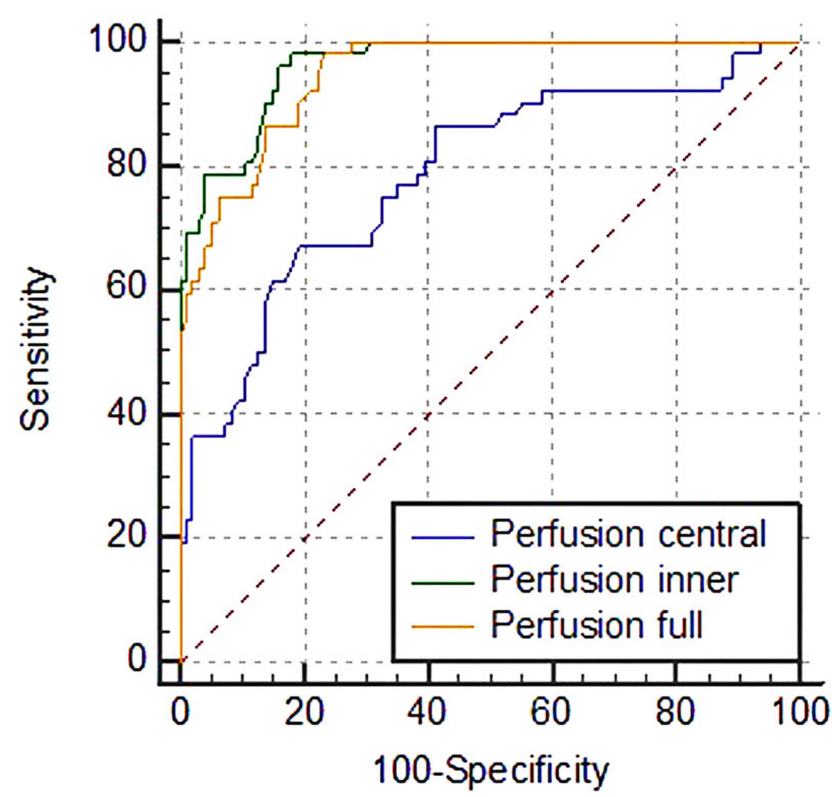

Figure $3 \mathrm{ROC}$ analysis for perfusions density reveals statistically significant difference between ROC curves for PD foveal and both PD parafoveal $(P<0.001)$ and PD total $(p<0.00 \mathrm{I})$, whereas there is no statistically significant difference between the ROC curves for PD parafoveal and PD total $(P=0.057)$. Both tests were highly discriminative.

When considering the patient-specific treatment schemes has gained importance in glaucoma management recently, the vascular parameters are gradually becoming important in terms of diagnosis, progression, and treatment algorithms especially in XFG, which is known to affect the vascular system more and cause increased vascular resistance. ${ }^{18-24}$ Even a comprehensive slit-lamp examination is sufficient to diagnose XFS, and XFG is a clinical diagnosis depends on detection of glaucomatous damage; in particular cases with XFS it is hard to determine whether the VF defect is related with glaucoma or related with other co morbidities. Yarmohammadi et $\mathrm{al}^{25}$ have found that that OCTA potentially shows promise for identifying focal glaucomatous damage before the first VF defect develops. OCTA can be used to determine vascular changes due to glaucoma, and differentiate changes between eyes with exfoliation. The vascular status of the patient's FAZ area may change the preferred treatment priority for the eyes with exfoliation.

There were only a few studies in the literature on FAZ area and macular VD at XFG measuring with OCTA. Magrath et $\mathrm{al}^{26}$ studied variability of capillary density in healthy subjects using 2 different machines and reported that the measurements taken with the same machine were consistent and reliable between fellow eyes, but there was variability exists among different 
machines. Philip et $\mathrm{al}^{27}$ found lower macular vascular density in XFG compared to POAG patients. They did not find any differences in FAZ parameters between $\mathrm{XFG}$ and POAG, possibly revealing similar damage at the central foveal region. They suggested that macular vessel density measurement may be useful in following the progression of glaucomatous patients with central field defects. They found more severe vascular involvement in XFG patients and suggested a different pathogenesis of damage that may correspond with the more severe nature of XFG. However, they did not examine a control group to determine the differences compared to healthy subjects. Rebolleda et $\mathrm{al}^{28}$ found decreased capillary density in glaucoma eyes compared to healthy eyes and reported significantly lower capillary density in XFG compared to POAG at similar glaucoma damage.

OCTA shows the early numerical finding of microvascular pathology in XFS. ${ }^{29}$ OCTA provides a parameter for, predicting the risk of glaucoma development in patients at risk of glaucoma, and assessing glaucomatous progression in patients with stable XFG according to foveal vascularity. ${ }^{29}$ They showed progressive decrease in peripapillary perfused capillary density (PCD) from controls to XFS to POAG to XFG. ${ }^{29}$ More significant decrease in PCD was shown in both XFG and XFS compared to POAG and healthy subjects, respectively. ${ }^{29}$ Suwan et $\mathrm{al}^{29}$ reported very important quantitative data of the microvascular disturbance in XFS, in peripapillary region.As a difference to what they did, we demonstrate decreased vascularity and perfusion in the foveal region. In addition, here we further showed the capability of OCTA as a diagnostic tool for detecting XFG, predicting the risk of glaucoma development, and assessing glaucomatous progression in patients with XFG by the help of cut-off values.

Impending glaucoma can be detected earlier by using the cut-off values we have found. OCTA helps to understand the vascular pathophysiologic changes in XFG and would be helpful in the follow up of exfoliative eyes which have higher GCC values, but which cannot be called as "progressing". Sayed et $\mathrm{al}^{30}$ reported that early preperimetric stages in which RNFL and GCC thicknesses are labelled green, can be missed by the clinician and may result in glaucoma being undetected in early glaucoma. Also Rao et $\mathrm{al}^{31}$ reported that OCTA has the capacity of differentiating early glaucomatous changes from healthy eyes and measurements also have the acceptable repeatability variability. There was also damage to the retina and choroidal vascular structures, reduced choroidal thickness and enlarged FAZ area in eyes with XFS. ${ }^{32}$ These changes in XFS becomes more noticeable as the disease progresses towards glaucoma and thereafter. Other ocular conditions that can increase retinal thickness may prevent diagnosis of co-existing glaucoma, also called "green disease". ${ }^{30}$ Since OCTA provides information about glaucoma patients at risk of high progression rate, it complements other traditional examinations, such as VF and OCT, not only to diagnose glaucoma but also to detect progression and assess the risk of progression. ${ }^{31}$

We revealed the foveal vascular features of the XFG and showed the differences in foveal vascular indices compared to normal controls. We aimed to extend our data by providing certain cut-off points for OCTA parameters in XFG patients. The ROC analysis reveals a clear separation differentiating those patients from healthy subjects.

Our study had some limitations. These cut-off values are relevant only for the device used in this study. It should be investigated whether these findings were also correlated in other types of devices. Because the patients were not followed over time, we could not reveal the disease progression. In the continuation of this study, we plan to study the differences in these cut-off values with other types of glaucoma. Further investigations by OCTA are needed for a better understanding the vascular changes in glaucoma patients.

\section{Conclusion}

The parameters VD, PD and FAZ have the capability to differentiate XFG from normal subjects, as their values are lower in XFG patients. Given the high sensitivity and specificity of reported cut-off values to differentiate XFG from healthy eyes, OCTA could be an important clinical tool in glaucoma management to show how far a particular exfoliative eye is positioned from a healthy eye.

\section{Abbreviations}

BCVA, Best corrected visual acuity; CST, Central subfield thickness; FAZ, Foveal avascular zone; FAZ, Foveal avascular zone; GCC, Ganglion cell complex; GCL+IPL, Ganglion cell layer-inner plexiform layer; IOP, Intraocular pressure; NTG, Normal tension glaucoma; OA, Ophthalmic artery; OCTA, Optical Coherence Tomography Angiography; PD, Perfusion density; POAG, Primary open-angle glaucoma; XFG, Pseudoexfoliation Glaucoma; XFS, Pseudoexfoliation syndrome; RI, Resistivity index; RGCs, Retinal ganglion cells; RNFL, Retinal nerve fiber layer; 
RNFs, Retinal nerve fibers; SITA-standard, Swedish Interactive Thresholding Algorithm; VD, Vessel density; VF, Visual field.

\section{Data Sharing Statement}

The datasets used and/or analyzed during the current study are available from the authors on reasonable request.

\section{Ethics Approval and Consent to Participate}

All procedures performed in studies involving human participants were in accordance with the ethical standards of the institutional and/or national research committee and with the 1964 Helsinki declaration and its later amendments or comparable ethical standards. This study was approved by the Ethics Committee of the Eye Hospital Clinic Maja with the approval number of Q11/10-2017. Written informed consents were obtained from all participants included in the study.

\section{Disclosure}

The authors report no conflicts of interest for this work.

\section{References}

1. Aasved H. Mass screening for fibrillopathiaepitheliocapsularis, so-called senile exfoliation or pseudoexfoliation of the anterior lens capsule. Acta Ophthalmol. 1971;49:334-343. doi:10.1111/j.1755-37 68.1971.tb00958.x

2. Ritch R. Exfoliation syndrome: the most common identifiable cause of open-angle glaucoma. J Glaucoma. 1994;3:176-178. doi:10.1097/ 00061198-199400320-00018

3. Weinreb RN, Leung CK, Crowston JG, et al. Primary open-angle glaucoma. Nat Rev Dis Primers. 2016;2::16067. doi:10.1038/nrdp. 2016.67

4. Detorakis ET, Achtaropoulos AK, Drakonaki EE, Kozobolis VP. Hemodynamic evaluation of the posterior ciliary circulation in exfoliation syndrome and exfoliation glaucoma. Graefes Arch Clin Exp Ophthalmol. 2007;245(4):516-521. doi:10.1007/s00417-006-0439-7

5. Yuksel N, Karabas VL, Arslan A, Demirci A, Caglar Y. Ocular hemodynamics in exfoliation syndrome and exfoliation glaucoma. Ophthalmology. 2001;108:1043-1049. doi:10.1016/S0161-6420(01) 00572-3

6. Martinez A, Sanchez M. Ocular haemodynamics in pseudoexfoliative and primary open-angle glaucoma. Eye. 2008;22(4):515-520. doi:10. 1038/sj.eye.6702676

7. Galassi F, Sodi A, Ucci F, Renieri G, Pieri B, Baccini M. Ocular hemodynamics and glaucoma prognosis: a color Doppler imaging study. Arch Ophthalmol. 2003;121(12):1711-1715. doi:10.1001/ archopht.121.12.1711

8. Satilmis M, Orgul S, Doubler B, Flammer J. Rate of progression of glaucoma correlates with retrobulbar circulation and intraocular pressure. Am J Ophthalmol. 2003;135(5):664-669. doi:10.1016/S00 02-9394(02)02156-6

9. Lee EJ, Lee KM, Lee SH, Kim TW. Angiography of the peripapillary retina in primary open-angle glaucoma. Invest Ophthalmol Vis Sci. 2016;57:6265-6270. doi:10.1167/iovs.16-20287
10. Mammo Z, Heisler M, Balaratnasingam C, et al. Quantitative optical coherence tomography angiography of radial peripapillary capillaries in glaucoma, glaucoma suspect, and normal eyes. Am J Ophthalmol. 2016;170:41-49. doi:10.1016/j.ajo.2016.07.015

11. Mansoori T, Sivaswamy J, Gamalapati JS, Balakrishna N. Radial peripapillary capillary density measurement using optical coherence tomography angiography in early glaucoma. J Glaucoma. 2017;26:438-443. doi:10.1097/JJG.0000000000000649

12. Shin JW, Lee J, Kwon J, Choi J, Kook MS. Regional vascular density-visual field sensitivity relationship in glaucoma according to disease severity. Br J Ophthalmol. 2017;101:1666-1672. doi:10.11 36/bjophthalmol-2017-310180

13. Yarmohammadi A, Zangwill LM, Diniz-Filho A, et al. Optical coherence tomography angiography vessel density in healthy, glaucoma suspect, and glaucoma eyes. Invest Ophthalmol Vis Sci. 2016;57: 451-459. doi:10.1167/iovs.15-18944

14. Yarmohammadi A, Zangwill LM, Diniz-Filho A, et al. Relationship between optical coherence tomography angiography vessel density and severity of visual field loss in glaucoma. Ophthalmology. 2016;123:2498-2508. doi:10.1016/j.ophtha.2016.08.041

15. European Glaucoma Society Terminology and Guidelines for Glaucoma, 4th edition - part 1 supported by the EGS Foundation. Br J Ophthalmol. 2017;101:1-72.

16. Mills RP, Budenz DL, Lee PP, et al. Categorizing the stage of glaucoma from pre-diagnosis to end-stage disease. $\mathrm{Am}$ J Ophthalmol. 2006;141(1):24-30. doi:10.1016/j.ajo.2005.07.044

17. Shields MB. Normal-tension glaucoma: is it different from primary open-angle glaucoma? Curr Opin Ophthalmol. 2008;19(2):85-88. doi:10.1097/ICU.0b013e3282f3919b

18. Kocaturk T, Isikligil I, Uz B, Dayanir V, Dayanir YO. Ophthalmic artery blood flow parameters in pseudoexfoliation glaucoma. Eur J Ophthalmol. 2016;26:124-127. doi:10.5301/ejo.5000671

19. Uz B, Durum Y, Kocatürk T, Dayanir YO, Dayanir V, Bilgen M. Carotid arterial flow in pseudoexfoliation glaucoma and its role in diagnosing the disease. J Glaucoma. 2016;25:959-962. doi:10.1097/ IJG.0000000000000546

20. Galassi F, Giambene B, Menchini U. Ocular perfusion pressure and retrobulbar haemodynamics in pseudoexfoliative glaucoma. Graefes Arch Clin Exp Ophthalmol. 2008;246:411-416. doi:10.1007/s00417-007-0709-Z

21. Dayanir V, Topaloglu A, Ozsunar Y, Keceli M, Okyay P, Harris A. Orbital blood flow parameters in unilateral pseudoexfoliation syndrome. Int Ophthalmol. 2009;29:27-32. doi:10.1007/s10792-008-9193-7

22. Kocaturk T, Isıklıgil I, Dayanir V, Ozsunar Y, Cakmak H. Ischemic changes in a case of unilateral pseudoexfoliation syndrome. J Clin Anal Med. 2016;7:87-89.

23. Yaşar E, NilgünYıldırım N, Atalay E. Pseudoexfoliation syndrome and antidepressant drug use. Turk J Ophthalmol. 2019;49(1):1-5. doi:10.4274/tjo.galenos.2018.06887

24. Schumacher S, Schlötzer-Schrehardt U, Martus P, Lang W, Naumann GO. Pseudoexfoliation syndrome and aneurysms of the abdominal aorta. Lancet 2001;357:359-360. doi:10.1016/S01406736(00)03645-X

25. Yarmohammadi A, Zangwill LM, Diniz-Filho A, et al. Peripapillary and macular vessel density in glaucoma patients with single-hemifield visual field defect. Ophthalmology 2017;124:709-719. doi:10.1016/j.ophtha. 2017.01.004

26. Magrath GN, Say EAT, Sioufi K, Ferenczy S, Samara WA, Shields CL. Variability in foveal avascular zone and capillary density using optical coherence tomography angiography machines in healthy eyes. Retina 2017;37:2102-2111. doi:10.1097/IAE.0000000000001458

27. Philip S, Najafi A, Tantraworasin A, Chui TYP, Rosen RB, Ritch R. Macula vessel density and foveal avascular zone parameters in exfoliation glaucoma compared to primary open-angle glaucoma. Invest Ophthalmol Vis Sci. 2019;60:1244-1253. doi:10. 1167/iovs.18-25986 
28. Rebolleda G, Pérez-Sarriegui A, De Juan V, Ortiz-Toquero S, Muñoz-Negrete FJ. A comparison of two optical coherence tomography-angiography devices in pseudoexfoliation glaucoma versus primary open-angle glaucoma and healthy subjects. Eur J Ophthalmol. 2019;29:636-644. doi:10.1177/112067211880 5882

29. Suwan Y, Geyman LS, Fard MA, et al. Peripapillary perfused capillary density in exfoliation syndrome and exfoliation glaucoma versus POAG and healthy controls: an OCTA study. Asia Pac J Ophthalmol. 2018;7:84-89. doi:10.22608/APO.2017318

30. Sayed MS, Margolis M, Lee RK. Green disease in optical coherence tomography diagnosis of glaucoma. Curr Opin Ophthalmol 2017; 28:139-153.
31. Rao HL, Pradhan ZS, Suh MH, Moghimi S, Mansouri K, Weinreb RN. Optical coherence tomography angiography in glaucoma. J Glaucoma. 2020;29:312-321. doi:10.1097/IJG.00000 00000001463

32. Çınar E, Yüce B, Aslan F. Retinal and choroidal vascular changes in eyes with pseudoexfoliation syndrome: a comparative study using optical coherence tomography angiography. Balkan Med J. 2019;37:9-14. doi:10.4274/balkanmedj.galenos.2019.2019.5.5

\section{Publish your work in this journal}

Clinical Ophthalmology is an international, peer-reviewed journal covering all subspecialties within ophthalmology. Key topics include: Optometry; Visual science; Pharmacology and drug therapy in eye diseases; Basic Sciences; Primary and Secondary eye care; Patient Safety and Quality of Care Improvements. This journal is indexed on PubMed

Submit your manuscript here: https://www.dovepress.com/clinical-ophthalmology-journal
Central and CAS, and is the official journal of The Society of Clinical Ophthalmology (SCO). The manuscript management system is completely online and includes a very quick and fair peer-review system, which is all easy to use. Visit http://www.dovepress.com/ testimonials.php to read real quotes from published authors. 